\title{
Subtle Changes of Basketball Player in Video Based Euler Method
}

\author{
Septian Enggar Sukmana*1, Nisa'ul Hafidhoh ${ }^{2}$ \\ Universitas Dian Nuswantoro / Informatics Department, Jalan Imam Bonjol 207 Semarang, \\ (+6224) 3517261/(+6224) 3569684 \\ E-mail : septian.enggar@dsn.dinus.ac.id*1,nisa@dsn.dinus.ac.id ${ }^{2}$ \\ *Corresponding author \\ Dwi Harini Sulistyawati ${ }^{3}$ \\ University of 17 Agustus 1945 Surabaya/Informatics Department, Jalan Semolowaru No. 45 \\ Menur Surabaya, (031) 5931800 \\ E-mail :dwiharini@untag-sby.ac.id ${ }^{3}$
}

\begin{abstract}
Analysing basketball player movement is not performed conventionally recently. Combining science and technology in sport has made a great impact. Basketball player movement analysis using latest technology can achieved by many ways such as retrieving information from video. A video that contains basketball match can be valuable resource to be analysed by computer vision technique. Player movement estimation can be separated into two categories such explicitly or implicitly. Player movement estimation that is performed implicitly is assumed more efficient than explicitly one, but in consequent, it has exploiting series computation like Lagrangian or Eulerian to the pixel color value and it is not easy to be seen by human visual system. This paper uses Eulerian series because of its succest in the previous research activity that called eluerian video magnification technique. That technique is implemented to basketball player in the field and it is not easy task because it is the object is formed as group in certain environment.
\end{abstract}

Keywords - basketball player movement, eulerian video magnification, subtle motion

\section{INTRODUCTION}

A precision in player position and movement in video after mapping is crucial to know (Fig. 1). That precision must be resulted from exact position and direction of movement at one player in the field. However, implementation of object detection based HSV and object detection based PCA still does not get better result for player movement that will be transformed into mapping. There is still missing detection on some players (Fig. 2). It must be a disadvantage because analysis of basketball playing using video processing can not be done properly. It is important to know because playing style of each player is a very important study material for a team who will play with another team. 


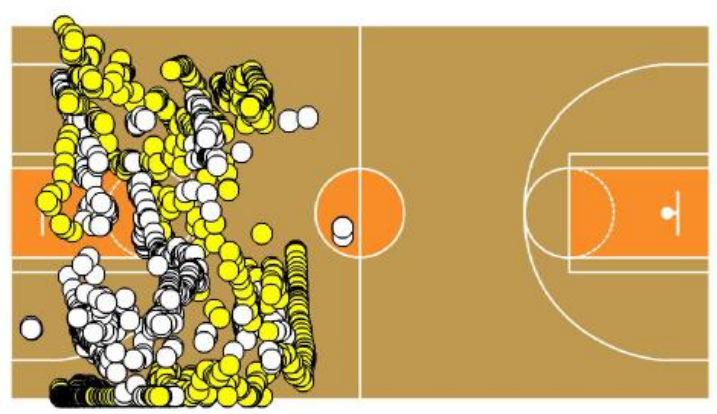

Figure 1. Sample of basketball player movement mapping

Analysing basketball playing using latest technology via video is important and can be one of main training components [9] especially for information how to beat another team before meet in the match. Analysis result can make a big impat to arrangging player position, starting player, substitution player [3].

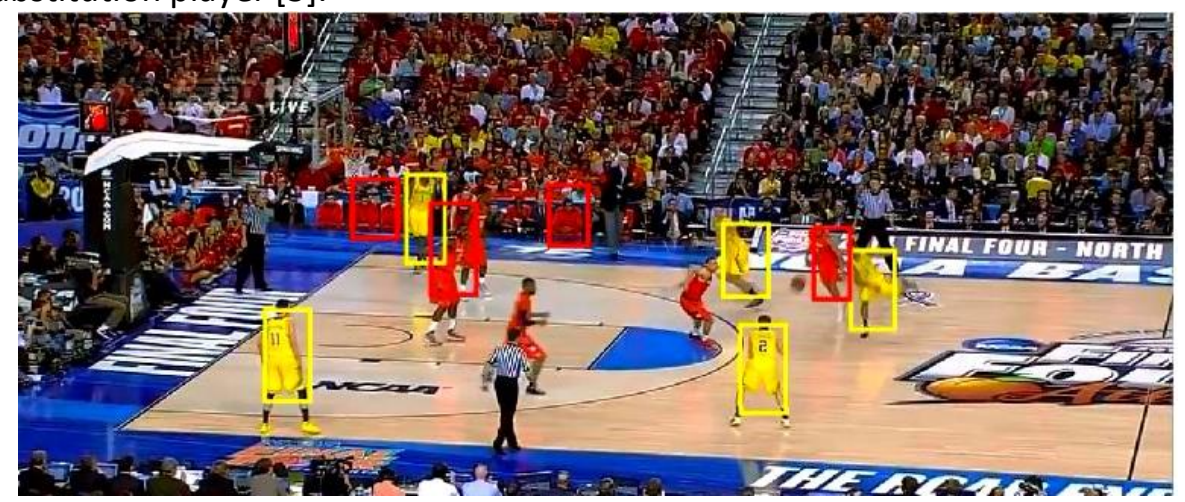

Figure 2. Some players are not detected properly

Moreover, implementation of the latest science and technology is also used to help better analysis like exploiting statistical model by combinatorial gaming theory to optimize player movement analysis [4]. Tao et.al [5] develop a computation of raw data to developed data based graph theory by link prediction approachment to give information about player movement in the field. Detection and tracking of a sport team is also developed by implementing computer vision technique [6].

Player movement is related to motion condition of that player. Object movement can be known by two ways, implementing movement estimation explicitly or implicitly [1]. But, implementation of movement estimation explicitly like example in paragraph above may need large computation resource [1] so data processing by implementation of movement estimation implicitly can be solution because its spatio-temporal processing is not burdened by accuration of movement estimation requirement factor.

However, spatio-temporal processing needs series computing in its spatial movement for that video. Pixel color value in video will be computed by its value changing, it can be an information about many phenomenas that happen including subtle motion that can not be seen by human visual.

There are two kinds of pixel color value computation using spatio-temporal processing suchimplementation of Lagrangian series [7-8] and implementation of Eulerian series [1-2] to observe subtle motion. Eluerian series is choosen by Wu et.al [1] and Wadhwa et.al [2] because more efisien in computation.

This paper shows implementation of Eulerian series in pixel color value that generated by Taylor series expansion or eulerian video magnification that developed by Wu et.al[1]. It is challenging because of the contribution in modernization of sport especially basketball and the 
impact eulerian video magnification technique to grouping object movement in certain environment.

\section{RESEARCH METHOD}

In this activity, the technique follows eulerian video magnification which starts from providing data, then spatial decomposition is applied to create different spatial band, after that temporal processing is performed with the same filter band to all bands which is created in the previous step, and processing result is added to original signal to get output video (Fig. 3). About contribution, this activity has purpose to get better result in basketball player movement mapping information by analysis its motion and color subtle and to create basic properties of player position and condition in the field. Data that is used in this activity is general, for testing video from YouTube is used. It is important to know that magnification technique consists of spatial decomposition and temporal processing, magnification technique uses magnification factor $\alpha$ that is very fully advantage to be standard parameter in amplification.

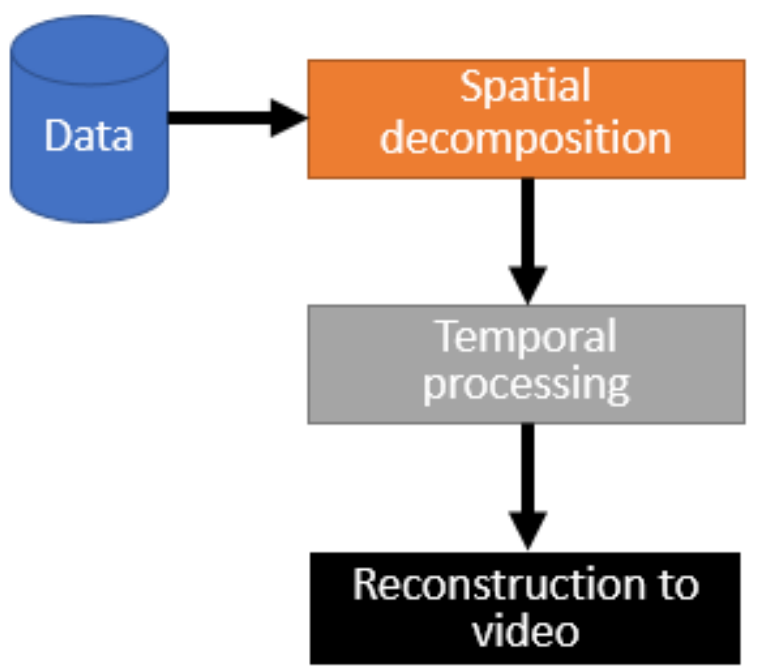

Figure 3. Basic Methodology's step

Motion and color subtle changes in video is related to combination between spatial and temporal processing. Because of that, decomposition of video sequence is necessary to get different spatial frequency bands. So, magnification technique is performed differently in each spatial band using same $\alpha$.

Motion magnification is produced by temporal processing using first-order Taylor series expansions so that synthesization of signal can be reached properly

$I(x, t)=f(x+(1+\propto) \delta(t))$

where $I(x, t)$ is image intensity at position $x$ and time $t$.

When an assumption about image is followed by first-order Taylor expansion, there are some modifcation in time $t$ about $x$

$I^{\prime}(x, t)=f(x)+\delta(t) \frac{\delta f(x)}{\partial x}$

Implementation result of broadband temporal bandpass filter $B(x, t)$ to the new assumption of image intensity will create

$B(x, t)=\delta(t) \frac{\delta f(x)}{\partial x}$

Amplification of bandpass signal uses $\alpha$, the it is added to the image intensity

$I^{\prime}(x, t)=I(x, t)+\propto B(x, t)$ 
The output will be created in the form of $I^{\prime}(x, t)$ approximation to $f(x+(1+\propto) \delta(t))$. It happens because of combination between (2), (3), and (4).

$I^{\prime}(x, t) \approx f(x)+(1+\propto) \delta(t) \frac{\delta f(x)}{\partial x}$

However, first-order Taylor approximation still produces a failure especially on image functions which changes quickly. So, the processed signal $I^{\prime}(x, t)$ must be equal to magnified function.

$f(x)+(1+\propto) \delta(t) \frac{\delta f(x)}{\partial x} \approx f(x+(1+\propto) \delta(t))(6)$

Let $f(x)=\cos \omega x$ for spatial frequency $\omega$ and $\beta=1+\alpha$ for (7) then use addition law for cosines (8).

$\cos (\omega x)-\beta \omega \delta(t) \sin (\omega x) \approx \cos (\omega x+\beta \omega \delta(t))(7)$

$\cos (\omega x)-\beta \omega \delta(t) \sin (\omega x)=\cos (\omega x) \cos (\beta \omega \delta(t))-\sin (\omega x) \sin (\beta \omega \delta(t))(8)$

\section{RESULTS AND DISCUSSION}

In this activity, there are three parameters that used for testing: $\alpha=20$ for motion amplification; $\alpha=50$ with four levels for color amplification; and $\alpha=50$ with six levels for color amplification. The video of match between Michigan and Minnesota is used for experiment. Each team can be identified from their jersey's color, the blue one or yellow one (fig. 4).

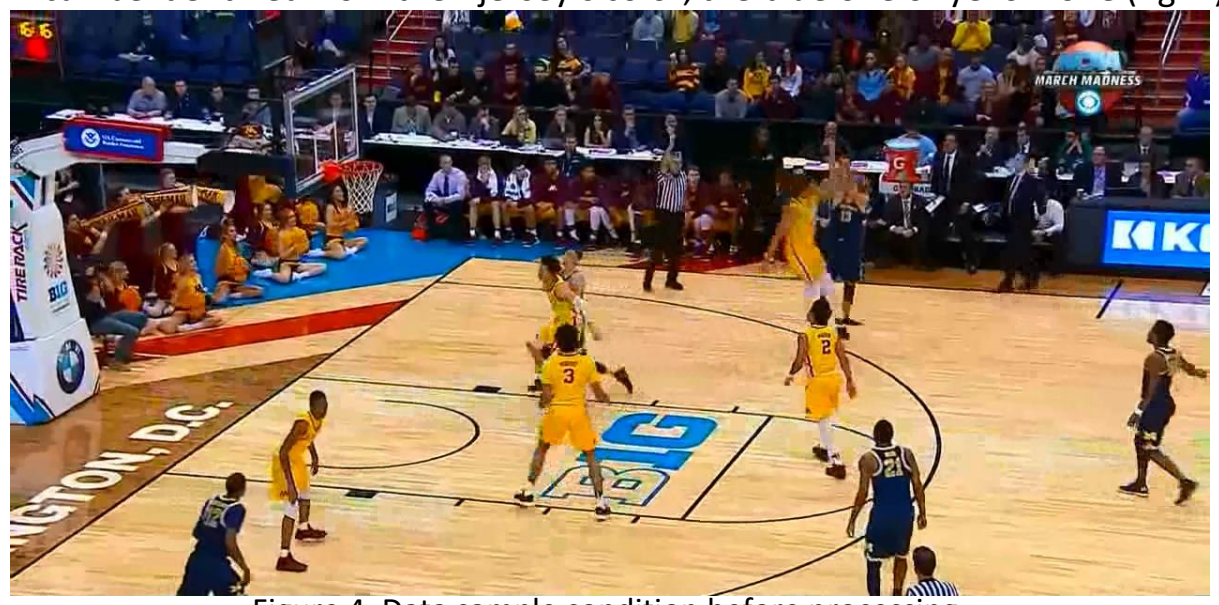

Figure 4. Data sample condition before processing.

Based on experiment, each $\alpha$ that is used show different subtle motion representation. By using $\alpha=20$, it tells that many of players that move in the field are detected properly (Fig. 5). It has been focused on each player so when a player who makes a move can be identified from player who is marked as black in the result, although in this result, there are some of motion player are body detected partially (red marked cirlce in Fig. 5). Focused on player, the result of motion magnification is quite high. Every black area in the field tells about each motion in every player. 


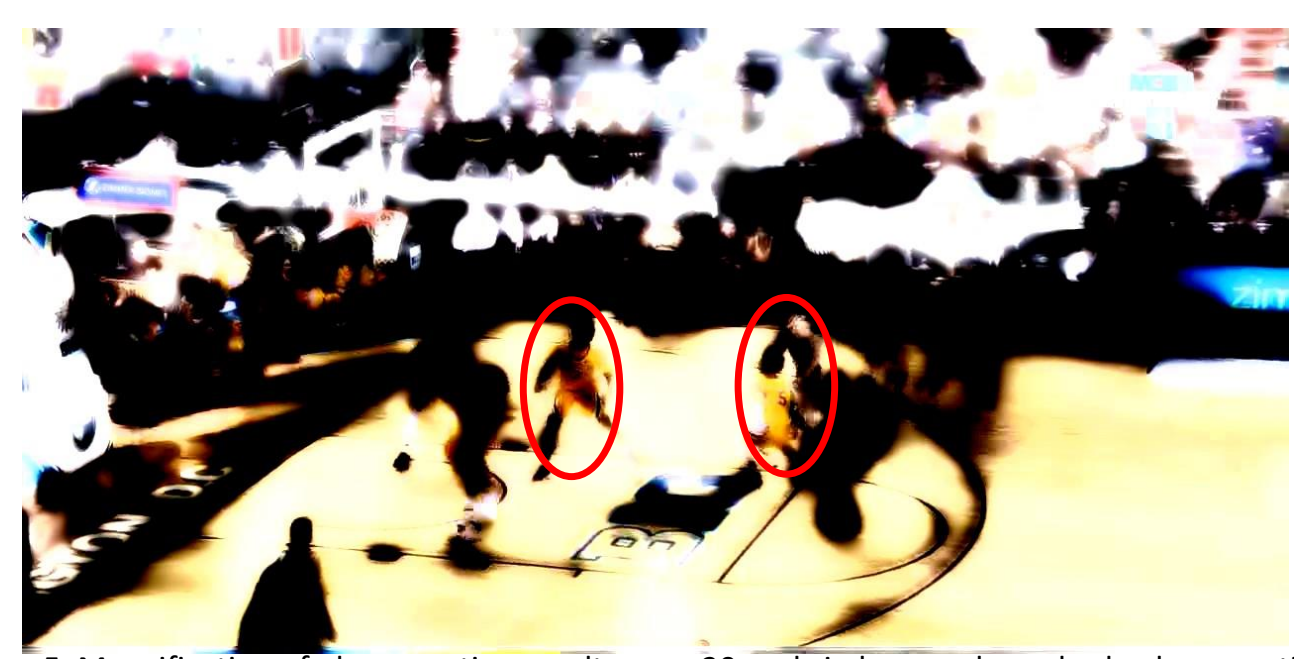

Figure 5. Magnification of player motion result on $\alpha=20$, red circle area shows body player motion is known partially.

Fig. 6 shows color magnification result using $\alpha=50$ with four spatial decomposition levels. Different from motion magnification, the color magnification result seems rather suitable for field condition than player condition. It can be identified that dominancy of color condition is more on field than player, or in other word the variation of color in the field area informs whether field is not positioned by player or is positioned by player partially. Although field condition is more dominant than player motion in color magnification representation, it is not true that player motion that represents as black area does not give effect on field condition. Fig. 6(a) shows when some players crowd part of field, it has more black area than less one (fig. 6(b)).

Beside field color representation, there is another color that appears in the field area and the blue one is the most explicit of all color. It is not surprised since jersey's color is also amplified. It tells that player with certain jersey's color is in that position but no explicit movement (this motion is too little to be magnified), or from previous moment there were a player but he has been long gone in the present frame.

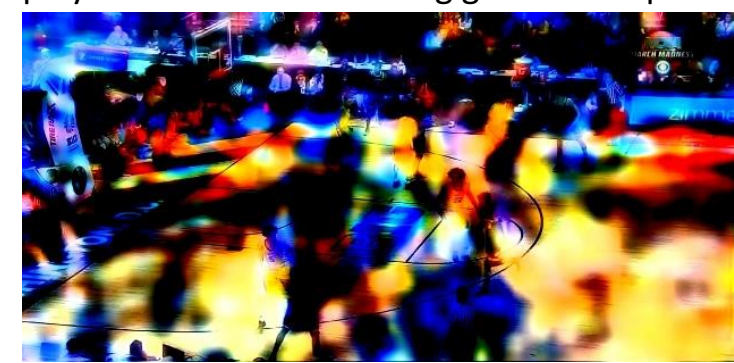

(a)

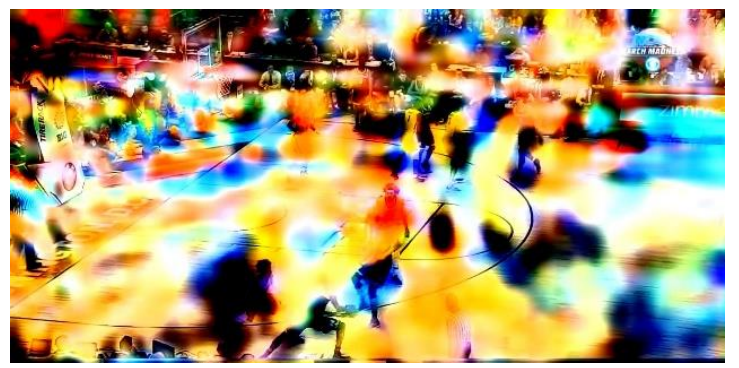

(b)

Figure 6. Color magnification result using $\alpha=50$ with four spatial decomposition levels: a) crowded condition; b) less player in the focused part of field.

Six decomposition levels result shows jersey color is more informable than field condition. Based on fig. 7, it shows that color amplification from jersey is more dominant than field one. Beside that, black area that represents as player motion is not appear stabily, black area appearing does not follow player motion regularly. 


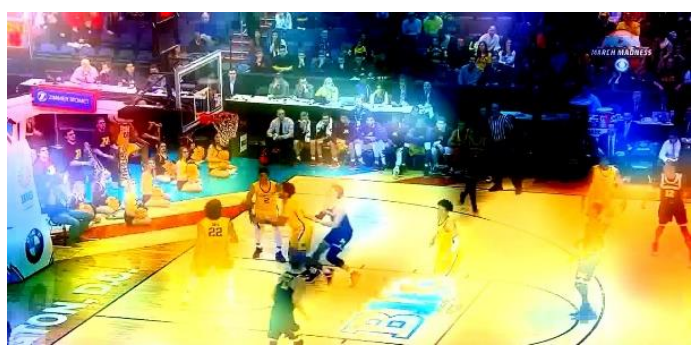

(a)

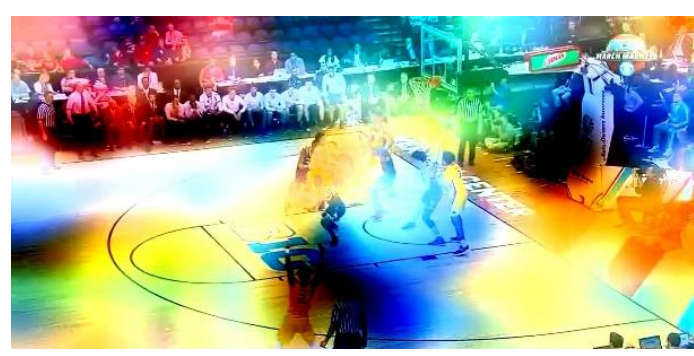

(b)

Figure 7. Color magnification result using $\alpha=50$ with six spatial decomposition levels: a) yellow jersey color amplification dominated; b) blue jersey color amplification dominated.

\section{CONCLUSION}

Implementation eluerian video magnification in basketball player in video has given some data properties that can be useful for mapping player such player subtle motion that can be useful for identification player movement and subtle color that can be useful for mapping position of player in the field. To be honest, it still has an unsatisfaction result which there is still some objects that cannot be identified properly and in color magnification using $\alpha=50$ with six spatial decomposition levels does not give better result in player subtle motion identification. For the next activity, it will exploit histogram of oriented gradients combined with support vector machine to get better player movement identification individually.

\section{REFERENCES}

[1] Wu H.Y, Rubinstein M, Shih E, Guttag J, Durand F, Freeman W. Eulerian Video Magnification for Revealing Subtle Changes in the World. ACM Transactions on Graphics-Siggraph 2012. 2012.

[2] Wadhwa N, Rubinstein M, Shih E, Guttag J, Durand F, Freeman W. Phase-Based Video Motion Processing. ACM Transactions on Graphics-Siggraph 2013. 2013.

[3] Sampaio J., McGarry T., Gonzalez J.C., Saiz J.S., Alcazar X.S., \& Balciunas M. Exploring game performance in the National Basketball Association Using Player Tracking Data. PLOS ONE. 2015.

[4] Kenter, F.H.J. An analysis of the basketball endgame: when to foul when trailing and leading. MIT Sloan Sports Analytics Conference. 2015.

[5] Zhang T., Hu G., \& Liao Q. Analysis of offense tactics of basketball games using link prediction. Electronics Library. 2013: 207-212

[6] Lu, W.L., Ting, J.A., Little, J.J., \& Murphy, K.M. Learning to track and identify players from Broadcast Sports Videos. IEEE Transactions on Pattern Analysis and Machine Intelligence. 2011.

[7] Wang J, Drucker S.M, Agrawala M, Cohen M.F. The Cartoon Animation Filter. ACM Transactions on Graphics-Siggraph 2006. 2006. 25: 1169-1173.

[8] Liu C, Torralba A, Freeman W.T, Durand F, Adelson E.H. Motion Magnification. ACM Transaction on Graphics. 2005. 24: 512-526.

[9] Theron R, Casares L. Visual analysis of time-motion in basketball games. $10^{\text {th }}$ International Symposium on Smart Graphics. 2010; 10: 196-207. 